\title{
A PIV-Guided Large-Eddy Simulation of In-Cylinder Flows
}

\author{
Franck Nicollet $^{1 *}$, Christian Krüger ${ }^{1}$, Jürgen Schorr ${ }^{1}$, Edouard Nicoud ${ }^{2}$, Olivier Colin ${ }^{2}$, \\ Christian Angelberger ${ }^{2}$, Johannes Bode ${ }^{3}$ and Benjamin Böhm ${ }^{3}$ \\ ${ }^{1}$ Daimler AG, HPC G206, 70546 Stuttgart - Germany \\ 2 IFP Energies Nouvelles, 1-4 avenue de Bois-Préau, 92852 Rueil-Malmaison Cedex - France \\ ${ }^{3}$ FG Energie- und Kraftwerkstechnik, Maschinenbau, TU Darmstadt, Jovanka-Bontschits-Straße 2, D-64287 Darmstadt - Germany \\ e-mail: franck.nicollet@daimler.com \\ * Corresponding author
}

\begin{abstract}
A combination of Large-Eddy Simulation (LES) and Particle Image Velocimetry (PIV) was utilized to investigate the three-dimensional in-cylinder flow within an optically accessible Direct Injection Spark Ignition (DISI) engine at motored engine operation. The PIV measurements were used to guide the meshing procedure by identifying the regions were refinements and improvements were needed. From the iteratively optimized meshes LES results are shown from two selected meshes, an intermediate coarse mesh and the final optimized mesh, and compared to PIV measurements. The evolution of the intake flow and the tumble in the central tumble plane during compression are presented and discussed. Exploitation of the LES results allowed showing the influence of out-of-plane velocities along the cylinder liner impacting the formation of the tumble flow. The optimized mesh was then used to investigate the influence of the spark plug on the incylinder flow. For the studied engine the spark plug had a significant impact on the evolution of the tumble flow during compression. Finally 35 engine cycles were simulated using the optimized mesh with the spark plug in place. Velocity distributions in a region below the spark plug are shown and compared with PIV results. The two-sample Kolmogorov-Smirnov test revealed a strong similarity between the velocity distributions obtained by PIV and LES, thus validating the potential of LES for investigating cycle-to-cycle variability.
\end{abstract}

\section{INTRODUCTION}

Strategies for lean combustion using stratified operation in the lower part load range with increasing homogeneous share for higher loads have a huge potential to reduce $\mathrm{CO}_{2}$ emissions. The main benefits are the result of improved de-throttling at low loads in combination with favorable gas properties and reduced wall heat losses under stratified engine operation.

In 2006, Mercedes-Benz introduced the spray-guided lean-burn combustion system to the gasoline engine, combining high specific engine output with reduced fuel consumption $[1,2]$. Future directions of development and research are the further reduction of $\mathrm{NO}_{\mathrm{x}}$ raw emissions by optimized EGR strategies in combination with an extension of the lean operation regime to higher loads. Those future targets increase even further the requirements for a robust and reproducible combustion process. The Mercedes-Benz spray-guided combustion system features a piezo actuated pintle-type injector (Bosch HDEV 4.1), a 200 bar fuel common rail and a spark-plug located between the exhaust valves. Different combustion modes are utilized: stratified (low load), homogeneous lean (mid load), and homogeneous stoichiometric (high load). The injector allows precise multiple injections even with very short needle opening times. Extensive measurements in closed vessels have 
shown the exceptional reproducibility of the fuel spray in terms of shape and stability. However during the stratified mode, high Cycle-to-Cycle Variations (CCV) of the incylinder flow lead to high fluctuations of the spray, fuel/air mixing and combustion [3]. As a result the robustness of the combustion is not ensured and the optimal engine mapping cannot be exploited, inducing higher fuel consumption and emissions and putting at risk the homologation of the car.

The stratified mode consists of three consecutive injections. The ignition takes place after the second injection. The work presented in $[4,5]$ has underlined the importance of the flow field located between the piston and the sparkplug directly before the first injection. It was demonstrated from high speed Particle Image Velocimetry (PIV) measurements that fluctuations of the flow in this region pointing upwards towards the spray led to strong fluctuations of the spray of the second injection. This "upward flow" was found to be amplified by the first injection. Furthermore this flow was found to correlate with Indicated Mean Effective Pressure (IMEP). For the stratified mode the evolution of the in-cylinder flow in the aforementioned region is therefore of great importance and needs to evolve very reproducible from cycle-to-cycle. The high speed PIV analysis gave new insights into the cause-and-effect chains and comprehensive datasets were build up in order to help further optimizing the engine for highly diluted engine operation.

PIV has been used extensively to capture in-cylinder flows [6] providing flow fields within a plane. High speed PIV provides crank-angle resolved flow fields and has been used to investigate cycle-to-cycle fluctuations $[7,8,9,10]$. To visualize the 3-Dimensional (3D)-flow Baum et al. [11] used tomographic PIV within few millimeter thick volumes surrounding the central tumble plane. Bode et al. [3] utilized a quasi-simultaneous multi-plane time-resolved PIV approach to investigate the flows evolution from the mid-valve plane into the central tumble plane. Despite recent improvements of PIV approaches flow field measurements are limited to plane or thin volumes and important regions of the flow as for example the flow in the intake port and the valve gap remain inaccessible.

Large-Eddy Simulation (LES) has been used to simulate in-cylinder flows for motored [12-14] and for fired engine operation where processes such as fuel injection, knock or emissions has been investigated [15-18]. LES gives access to the temporal evolution of the flow field in the entire flow domain. However, LES resolves only the largest flow scales of the instantaneous flow and needs sub-grid models in order to model the effects of the smallest scales. The threshold between the computed and the modelled scales is linked to the mesh cell size. As wall boundary layers cannot be resolved on practical meshes, "wall functions" are used to account for their effect. In view of the underlying assumptions, LES requires validation in order to check their reliability in view of a future usage in engine development processes. Many groups have validated their simulations of motored in-cylinder flows using PIV measurements $[12,14,19,20]$. The validation is typically based on the comparison of velocity profiles along selected lines. Janas et al. [13] have extended this analysis by comparing the evolution of the tumble vortex center during compression. For a more complete understanding of the differences between PIV measurements and LES other metrics as for example a Proper Orthogonal Decomposition (POD) or Relevance Index (RI) have been proposed [19]. All presented comparisons showed overall good or at least reasonable agreement whereas differences were observed locally. Even though these differences are small, they might become crucial since a correct prediction of flow features as the intake flow for example are of utmost importance to capture the global in-cylinder flow correctly [19].

Predictive simulations aim at capturing in-cylinder processes of new engine designs correctly without any input from experiments. In this sense LES is not predictive yet and needs validation first before it can be used for engine development. There is no universal reliable LES validation strategy available and the question remains open on which level of detail a validation is required. Depending on the process of interest different quantities for comparison as well as different level of agreement might be needed. The objective of this paper is to present a new validation strategy where high speed PIV has been used first to identify the relevant quantities and regions of the flow important for engine development and then validate the simulation by direct comparisons.

The aim of this ongoing effort is to build up a reliable LES dataset, complementary to the already existing PIV measurements. By combining the strengths of numerical simulations and experiments $\mathrm{CCV}$ can be analyzed in more detail providing new valuable insights useful to improve engine design. This paper presents the LES results of the motored in-cylinder flow together with a comprehensive validation. The PIV measurements were directly used to guide the meshing procedure. The mesh was iteratively optimized and the PIV measurements helped to identify the regions were further improvements were needed. A previous validated Reynolds Averaged Navier-Stokes (RANS) simulation of this configuration helped additionally to understand interaction of different 3D-flow structures responsible for the evolution of the tumble flow within the central tumble plane [4]. From this comprehensive mesh study two selected meshes are shown and discussed. Important flow features responsible for $\mathrm{CCV}$ as identified in previous work from PIV measurements were used for validation. For an accurate comparison between LES and the PIV measurements, a standard post-processing tool was developed allowing 


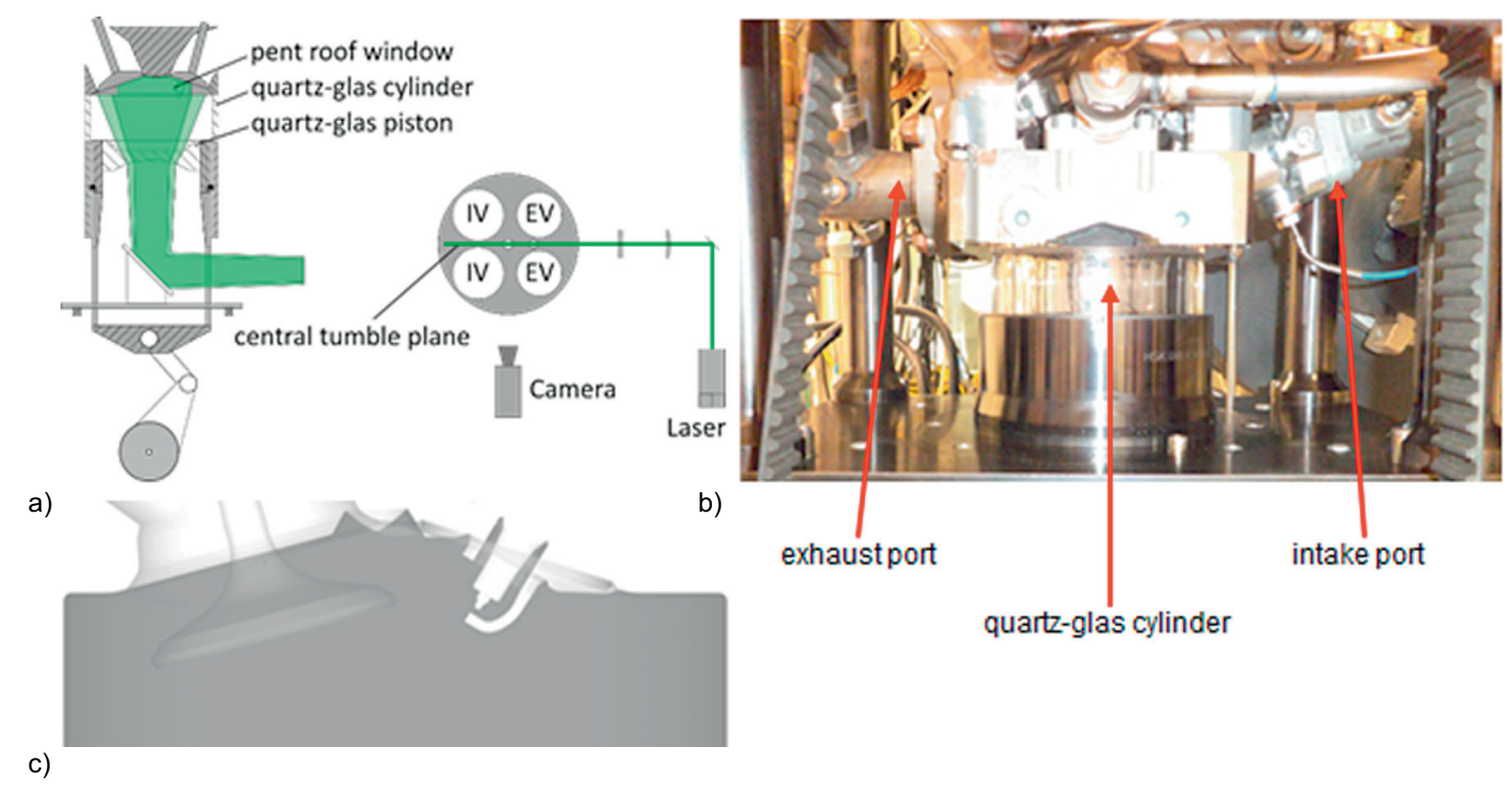

Figure 1

a) Experimental set-up: schematic side and top views of the optical engine with a single plane PIV system, b) side view of the optically accessible engine and c) 2D-view of the engine central tumble plane.

a similar post-processing and visualization of the experimental and numerical results. Since the spark plug is often removed in studies of the motored engine flow and was not part of this comprehensive mesh study an additional comparison of the flow with and without the spark plug was performed. Finally the cyclic variation of the "upward flow" which was identified in previous work to be responsible for CCV of the stratified combustion mode was compared to PIV measurements.

\section{EXPERIMENTAL SETUP AND METHODS}

The experiments and simulations were carried out on an optically accessible single-cylinder Direct Injection SparkIgnition (DISI) engine equipped with a four-valve pent-roof cylinder head. The engine features a centrally mounted injector and the spark plug was placed between the exhaust valves in the central tumble plane. Optical access was enabled by a quartz glass cylinder with a height of $35 \mathrm{~mm}$ and two pent-roof windows on both sides of the engine (Fig. 1). The piston in Bowditch arrangement was equipped with a window and featured a piston bowl. The engine characteristics and the operating point investigated are summarized in Table 1.

For the time resolved 2-Dimensional 2-Component (2D2C) PIV measurements a dual cavity frequency doubled $\mathrm{Nd}: \mathrm{YVO}_{4}$ laser (Edge wave IS120, $532 \mathrm{~nm}, 1 \mathrm{~mJ} /$ pulse) was used. The light sheet was focused with cylindrical lenses
TABLE 1

Engine characteristics and operating conditions.

\begin{tabular}{c|c}
\hline & Engine data \\
\hline Bore & $83 \mathrm{~mm}$ \\
\hline Stroke & $92 \mathrm{~mm}$ \\
\hline Compression ratio & 9.5 \\
\hline Engine speed & $2000 \mathrm{rpm} /$ motored \\
\hline Intake manifold pressure & $950 \mathrm{mbar}$ \\
\hline Intake valve opening (at 2 mm valve lift) & $35 \mathrm{aTDC}$ \\
\hline Coolant temperature & $200 \mathrm{aTDC}$ \\
\hline Oil temperature & $80^{\circ} \mathrm{C}$ \\
\hline
\end{tabular}

$(f=500 \mathrm{~mm})$ to $0.5 \mathrm{~mm}$ (Full Width at Half Maximum (FWHM)) thick light sheets. A concave cylindrical lens $(f=-50 \mathrm{~mm})$ together with a concave cylindrical lens at the bottom of the piston window generated a divergent light sheet enlarging the field-of-view. Silicon oil droplets $(\sim 0.5 \mu \mathrm{m}$ mean diameter) were seeded into a large plenum volume prior to the intake port to ensure homogeneous seeding densities using a cyclone based droplet seeder (Palas AGK 2000). A 12 bit CMOS camera (Vision Research Phantom v1610 $1280 \times 800$ active pixel) was used to record 
the scattered light in the central tumble plane. A variable pulse separation " $\mathrm{d} t$ " was optimized for the velocity scale of each phase. Two datasets were taken. For the first dataset (Campaign1) the spark plug was removed. The measurements were performed during intake and compression stoke from $360^{\circ}$ bTDC (before Top Dead Centre) up to TDC. At $2000 \mathrm{rpm}$ a double image was captured every $1.5^{\circ} \mathrm{CA}$ and 200 consecutive cycles were recorded. The second dataset (Campaign2) was taken with the spark plug in place. The measurements were performed in the compression stroke from $105^{\circ} \mathrm{bTDC}$ up to TDC. At $2000 \mathrm{rpm}$ a double image was recorded every $3^{\circ} \mathrm{CA}$ and 300 consecutive cycles were taken.

The PIV post processing was carried out with the commercial software Davis 8.2.2 (LaVision). To eliminate phase-steady artefacts, a background image was calculated by taking the minimal appearing intensity over all cycles for each pixel. This is done for each recorded ComputerAided Design (CAD) and the respective background image is subtracted from each image. Afterwards, an $8 \times 8$ pixel sliding Gaussian background subtraction and a local intensity normalization $(5 \times 5$ pixels $)$ was applied. The vector fields were calculated with a decreasing interrogation window size from $64 \times 64$ to $32 \times 32$ pixels and an overlap of $75 \%$. Important parameters of the PIV images are presented in Table 2. Vectors with a peak ratio below 1.7 were removed. Further inaccurate vectors were identified by neighbor comparison of the median and replaced by vector choices of higher order. Finally, local noise in the order of the spatial resolution was reduced with a $3 \times 3$ top hat filter.

\section{NUMERICAL SETUP AND METHODS}

AVBP [21] was used to perform LES. AVBP solves the compressible multi-species reactive Navier-Stokes equations on unstructured grids using an explicit time advancement. Convection is solved for using 2 nd and 3rd order schemes in space and time, in an attempt to limit a much as possible numerical dissipation. AVBP exhibits excellent speed-ups on parallel machines which is of particular interest for LES of piston engine flows since multiple cycles have to be computed with Computational Fluid Dynamics (CFD) meshes being more than 10 times larger than typical RANS's ones.

\subsection{CFD Model and Setup}

The computational domain includes the complete optically accessible engine geometry comprising the intake plenum and the exhaust port as shown in Figure 2. The piston crevices were not taken into account in the present study.
TABLE 2

Parameters of the PIV measurements.

\begin{tabular}{c|c|c}
\hline & $\begin{array}{c}\text { Flow field without } \\
\text { spark plug } \\
\text { (Campaign1) }\end{array}$ & $\begin{array}{c}\text { Flow field with } \\
\text { spark plug } \\
\text { (Campaign2) }\end{array}$ \\
\hline Recorded CAD & $360^{\circ}-0^{\circ}$ CA bTDC & $105^{\circ}-0^{\circ}$ CA bTDC \\
\hline Number of cycles & 200 & 300 \\
\hline Vector spacing & $0.65 \mathrm{~mm}$ & $0.65 \mathrm{~mm}$ \\
\hline Final interrogation area & $2.6 \mathrm{~mm}$ & $2.6 \mathrm{~mm}$ \\
\hline Field-of-view & $83 \times 45 \mathrm{~mm}$ & $83 \times 45 \mathrm{~mm}$ \\
\hline
\end{tabular}

The large penetration of the spark-plug in the combustion chamber leads to very strong interactions with the incoming intake flow and induces strong flow fluctuations in this region. The present study concentrates first on the global flow structures in the cylinder without the sparkplug geometry and secondly on the flow fluctuations including it. Thereby two CFD models were generated. The first CFD model (Model1) did not include the sparkplug geometry in order to be consistent with the PIV measurements of Campaign1. However the second CFD model (Model2) encompassed the detailed spark-plug geometry (electrodes and inner volume) as in Campaign2 (Fig. 2). A local mesh refinement of $0.3 \mathrm{~mm}$ was used within the spark-plug region.

The time step for the explicit time advancement of AVBP is driven by the smallest cell volume in the domain. Figure 3 shows the evolution of the minimum spatial resolution over $\mathrm{CAD}$ during the intake and the compression strokes. It remains relatively constant except for small valve lifts where a higher mesh resolution is needed in the valve curtains. Special care needs to be taken on the control of the minimum cell volumes especially during mesh deformation to ensure a high quality mesh. As a result the mesh generation using the software CENTAUR turned out to be a crucial but very time consuming procedure. An automated mesh generation process has been recently developed for AVBP and has drastically reduced the overall meshing time. In order to cover one engine cycle, several meshes have to be generated. The simulation runs using successively those meshes, deform them and map the final solution onto the next one. During the mesh deformation several cell quality criteria have to be fulfilled regarding the cell sliver, minimum cell volume etc. to ensure a robust run of the LES.

A mesh study was performed addressing separately the different engine parts. It was found that the global flow structures and the resulting tumble flow rotation speed were particularly dependent on the mesh resolution. 
a)

c)

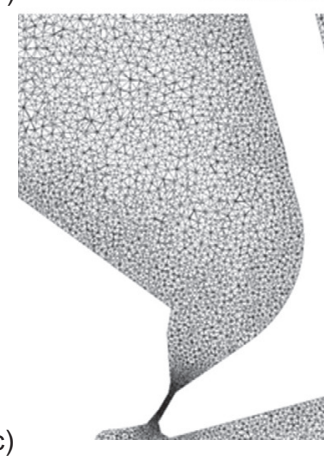

b)

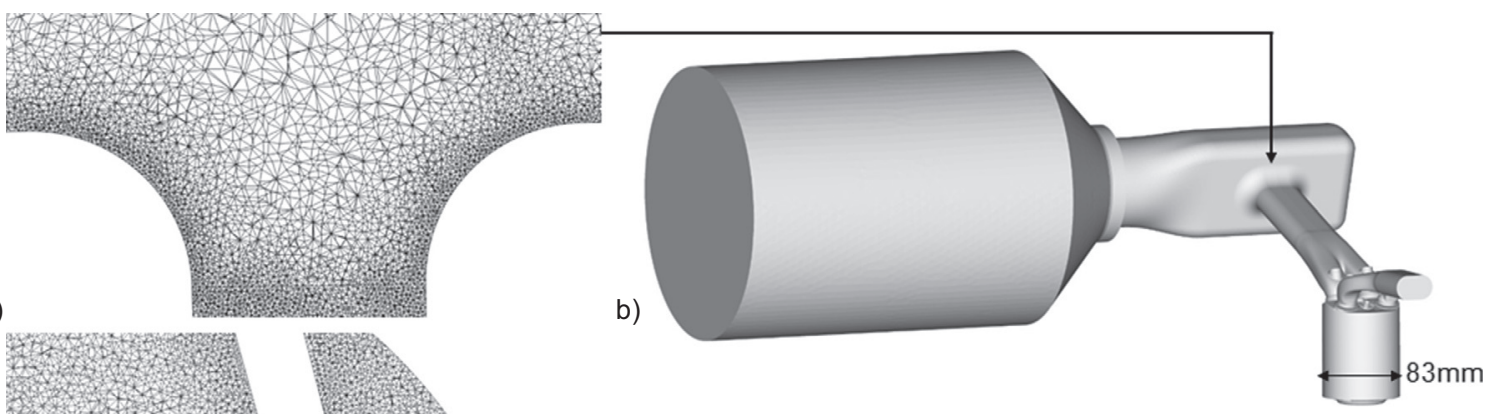

Figure 2

a) Mesh at the interface between the plenum and the intake port, b) CFD domain, c) mesh in the valve region (Model1_fine), d) mesh in the valve curtain region (Model1_fine), e) mesh in the spark-plug/injector region (Model2).

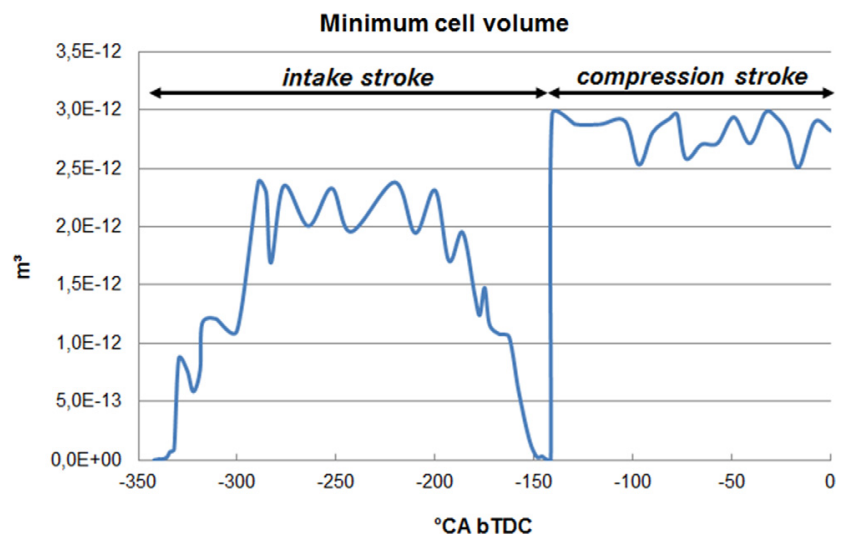

Figure 3

Minimum cell volume in the intake and compression stroke in Model2.

Therefore a particular attention was put on the setup of the mesh during the entire engine cycle. The overall mesh size, the adequate definition of the zones where the mesh needed to be refined, as well as the size of the cells at the walls have been addressed separately for each engine component. During the mesh study a multi-cycle LES run was performed (between 5 and 12 cycles) for every cell size change, analyzed and compared with the high speed PIV measurements. This allowed to converge iteratively (15 iterations) towards a robust CFD model reproducing the experimental results over the entire engine cycle.
The strategy followed during the mesh study can be summarized as follows:

- the maximal cell size at the walls was first iteratively derived based on the local $Y+$ values at the wall during the simulation to stay within the wall law validity domain $(Y+<100)$. The smallest cell sizes at the walls are located in the regions where the flow velocities is the highest (bottom part of the intake port, valves and upper part of the combustion chamber). All regions were investigated even the plenum and in the transition region between the plenum and the intake manifold which is often neglected (Fig. 2). The local turbulent to molecular viscosity ratio was used as indicator for the first mesh refinement in the regions of high flow velocities. This criterion limits the influence of the subgrid model in the regions where the flow has to be well captured. The target ratio was set to be $<50$,

- a mesh variation was then performed in the regions known to influence the flow: valves, valve curtains, intake port, cylinder, combustion chamber and piston. In this work the piston and the cylinder regions were always meshed in the same way. Each engine part is made of multiple CAD panel geometries. The meshing set-up consisted of four control parameters: the base cell size and the closest distance between the panels for the surface mesh and the base cell size and the growth ratio for the volume mesh. The mesh refinement was exclusively driven using those control parameters and not using "geometric source shapes" (cylinder, sphere and box). 
Indeed, the "geometric source shapes" were found unsuitable for local mesh refinement in combination with a complex engine geometry. Firstly it is difficult to position them locally and aligned to the 3D surface geometry; secondly the number of cells generated in those volumes is often too high compared to what is really needed. Each average LES result was compared to the PIV measurements to get an overall understanding of the local mesh influence on the LES results,

- the regions of minimal cell size were always monitored because they determine the overall time step of the simulation with the explicit solver. Incremental improvements of the mesh was achieved either by adjusting the meshing parameters in CENTAUR or by re-discretizing the surfaces in the CAD model.

The present study allowed us identifying the necessary mesh requirements for LES to yield an accurate reproduction of the PIV measurements. The final best practice mesh setup was derived (Modell fine in Tab. 3) and includes the following features. The total amount of tetrahedral elements ranges from 20 million@TDC to 44 million@BDC and the cell size was $0.35-0.5 \mathrm{~mm}$ in the intake port and cylinder. At valve opening and closing the cell size within the valve curtain was $60 \mu \mathrm{m}$. A set of 120 meshes is required to cover one engine cycle.

In the next sections of the paper the influence of the mesh size on the mean flow field will be presented. Therefore the results of a selected CFD model (Model1_coarse) from the iteratively mesh improvement procedure will be compared to the final mesh Model1_fine. Within Model1_coarse the cell size in the cylinder was $0.75 \mathrm{~mm}$ instead of $0.5 \mathrm{~mm}$. The cell size at the wall was kept the same to preserve wall friction and to enable a fair comparison between the two meshes. Furthermore the cell size in the vicinity of the intake valves was in the range of $0.35-0.5 \mathrm{~mm}$ instead of $0.35 \mathrm{~mm}$.

The in-/outflow boundary conditions were taken from measurements recorded during the experimental campaigns of the motored engine operation at the rate of $2000 \mathrm{rpm}$ and $P_{\text {int }}=950$ mbar. On the intake side a constant static pressure $\left(P_{\text {int }}=950 \mathrm{mbar}\right)$ was applied at the entrance of the plenum using a Navier-Stokes Characteristic Boundary Conditions (NSCBC) method to allow outgoing waves to leave the computational domain with minimum reflexions. The same pressure value was used to initialize the flow field in the CFD domain. On the exhaust side a transient static pressure profile was applied at the exhaust pressure sensor position located $38 \mathrm{~cm}$ downstream from the exhaust valve seats, also using NSCBC. The first two LES cycles were considered as "initialization cycles" and were not taken into account for the flow analysis.

Regarding the numerical setup of the simulation, a new No-Slip Wall Law (NSWL) presented in [22], combined with a second order Lax-Wendroff convective scheme and
TABLE 3

Mesh descriptions.

\begin{tabular}{c|c|c}
\hline & Model1_coarse & Model1_fine \\
\hline Number of cells & $12-23 \times 10^{6}$ & $20-44 \times 10^{6}$ \\
\hline Cell size int. valve & $0.35-0.5 \mathrm{~mm}$ & $0.35 \mathrm{~mm}$ \\
\hline Cell size valve curtain & $0.06-0.5 \mathrm{~mm}$ & $0.06-0.5 \mathrm{~mm}$ \\
\hline Cell size cylinder & $0.5-0.75 \mathrm{~mm}$ & $0.5 \mathrm{~mm}$ \\
\hline
\end{tabular}

the Smagorinsky subgrid scale turbulence model were used. An acoustic CFL value of 0.9 was used to limit the time step throughout the simulation. The resulting time step of Model1_fine was within $1.7 \times 10^{-8}$ to $2.5 \times 10^{-7} \mathrm{~s}$. The simulation took around 1.2 days per cycle on 450 cores. During the mesh study and the validation of the mean flow field with Model1, 12 consecutive cycles were run. For the validation of the cyclic flow velocity variations with Model2, the number of consecutive cycles was increased to 35 .

\section{LES VALIDATION}

\subsection{Validation Criteria for the Mean Flow Field}

For a reliable validation, flow structures which are relevant for the process of interest need to be identified first. From previous work the "upward flow" above the piston (Fig. 4c) in the central tumble plane was identified to be linked to cycle-to-cycle fluctuations of the spray and IMEP. Beside the importance of the central plane for ignition and subsequent combustion the tumble formation within this plane was found in previous RANS simulations [4] to be sensitive to the 3D out-of-plane flows. A characterization of these 3D flow structures and their mutual interactions is needed to understand the tumble flow's evolution. Figure 4a shows the sketch of the large flow structures identified during intake and compression strokes [4]. The flow distribution in the valve gap is responsible for the resulting in-cylinder flow motion. The inclined intake port together with an edge on the side of "flow number 2" results in a stronger "flow number 1" leading to a tumble flow motion which is typical for such engines [23]. After almost one revolution of "flow number 1" the flows leading edge "tumble front" begins to interact with "flow number 2". While "flow number 4" follows somehow the tumble flow within the central plane "flow number 3" is deflected by the cylinder walls towards "flow number 1". It moves along the cylinder wall and continues between the incoming "flow number 1" and the cylinder liner. Depending on the strength and direction of "flow number 3" the formation of the tumble can be shifted significantly leading to a different position of the tumble vortex. 


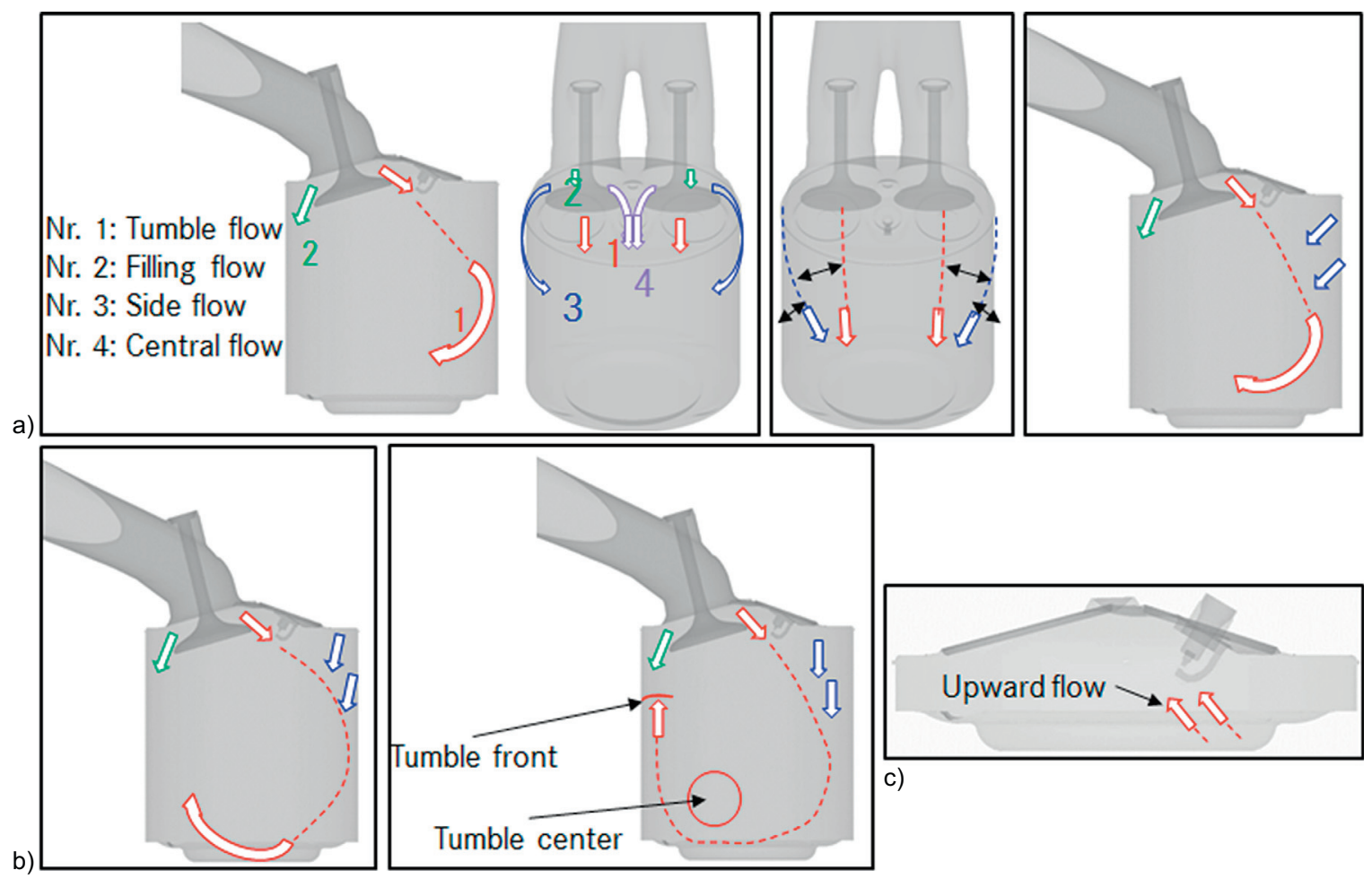

Figure 4

a) Main in-cylinder flows and representation of the primary and secondary flow interactions, b) tumble front/center and c) upward flow.

Already small changes of the velocity magnitude or direction of "flow number 3" can cause significant changes of the intake flow over the valve ("flow number 1") which dominates the evolution of the tumble vortex. These identified flow features have to be captured correctly in order to get the correct tumble flow rotation in the cylinder. Therefore for validating the LES the mean flow field was used in a first step to compare the evolution of the main flow structures within the central tumble plane with PIV measurements. The focus was on following flow structures (Fig. 4):

- evolution of "flow number 1 " identified by its leading edge ("tumble front") during intake,

- interaction of "flow number 1" and "flow number 2",

- the intensity and time of appearance of the "upward flow" at $40^{\circ} \mathrm{CA}$ bTDC (a typical timing for injection for stratified combustion),

- tumble center position during compression.

\subsection{Standard Post-Processing Tool}

An in-house standard post-processing tool has been developed over the last years in order to analyze and display the measurement and simulation results in the exact same way. The LES and PIV velocity fields were therefore mapped onto a common 2D-Cartesian grid of $0.5 \mathrm{~mm}$ cell size first.
The same Matlab routines were then applied to the LES and PIV data. This procedure ensures that exactly the same processing steps and definitions of the quantities of interest are applied to both the simulations and the experimental results.

\subsection{Validation of the Mean Flow}

This section presents the comparison of the mean flow field from LES and PIV measurements. The aforementioned flow structures will be discussed for the two selected meshes: The coarser mesh Model1_coarse and the final mesh Model1_fine. Figures 5-8 show the phase-averaged magnitude of the $n$-plane velocity component. The velocity vectors are overlaid on the PIV measurements to indicate the flow direction. The dotted horizontal line on the LES figures represents the bottom part of the optically accessible flow regions for PIV.

Figure 5 shows the flow in the central tumble plane during intake at $250^{\circ} \mathrm{CA}$ bTDC where the velocity distribution is characterized by a high velocity annular flow induced from the valves and the downward moving piston. The majority of the flow enters the cylinder through the valve gap over the front side of the valve leading to a strong "flow number 1 " which moves towards the cylinder liner and forms the clockwise tumble flow. A stagnation region is found 

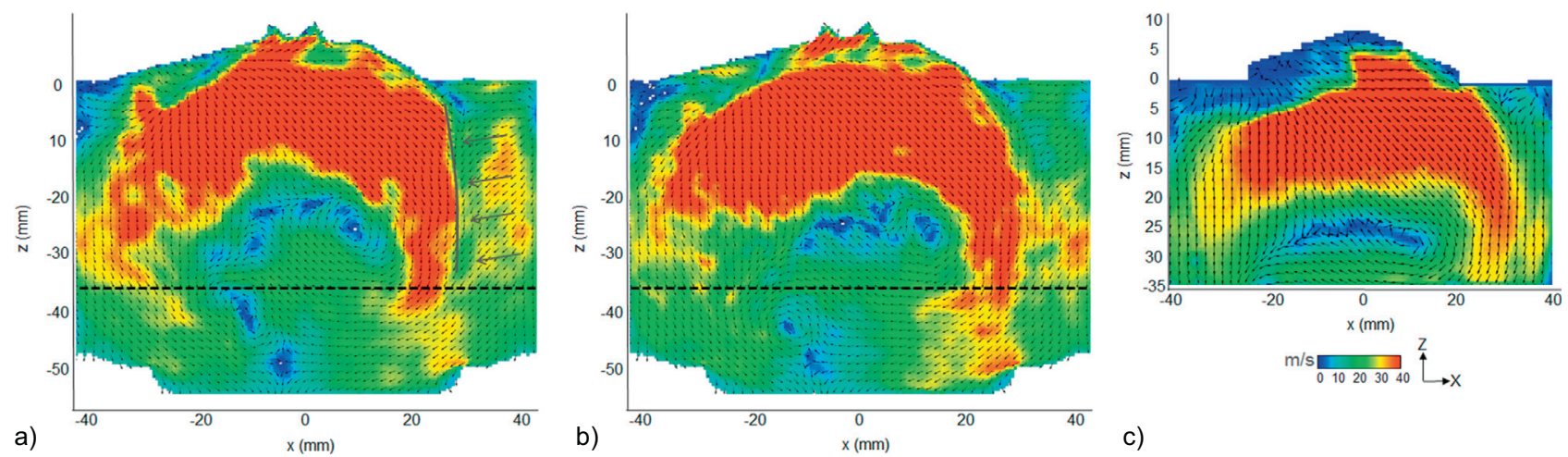

Figure 5

Average in-plane velocity magnitude $\left(V_{x z}\right)$ in the central tumble plane @ 250 CA Btdc: LES results averaged over 12 cycles (a) Model1_coarse, b) Model1_fine). c) High-speed PIV results averaged over 200 cycles (Campaign1) every (3rd 4th) vector shown.
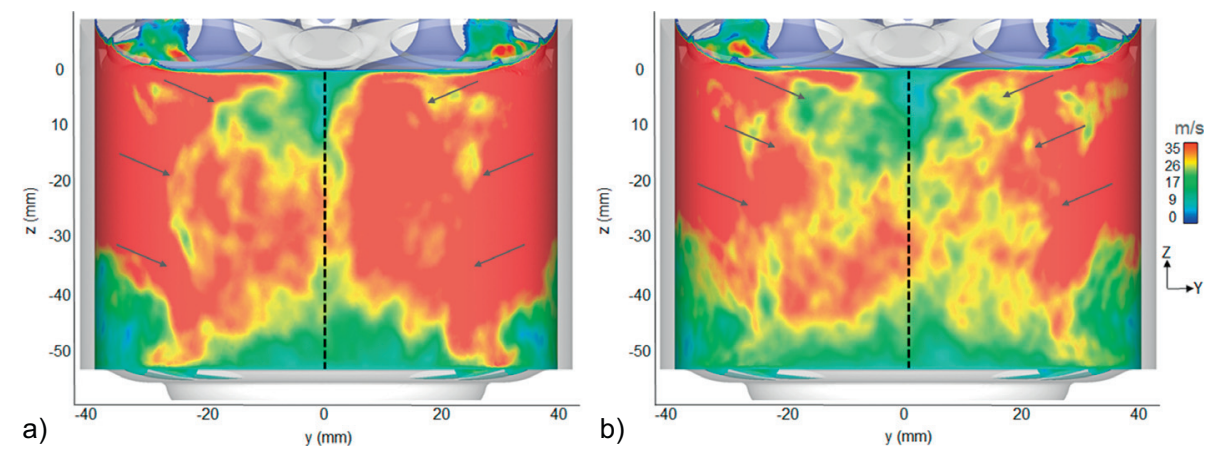

Figure 6

Average velocity magnitude $\left(V_{\mathrm{mag}}\right)$ along the cylinder surface @ radius=39 mm @ 250 CA bTDC. LES results averaged over 12 cycles (a) Model1_coarse, b) Model1_fine).

where the incoming flow impinges on the flow reversed by the piston left of the tumble vortex center which already begins to form. The LES results give access all the way to the cylinder walls and reveal a lower velocity region between "flow number 1" and the cylinder liner strong enough to keep "flow number 1" away from the liner and redirect it downwards towards the piston. This flow originating from the outer periphery of the intake valves, is redirected by the cylinder walls and travels along the cylinder all the way into the central plane and is labeled as "flow number 3". Figure 6 shows "flow number 3" from both intake valves along a circumferential surface located at $2.5 \mathrm{~mm}$ from the cylinder liner revealing how they impinge on each other in the central tumble plane, are redirected downwards towards the piston and form a stagnation region. The formation of the overall tumble flow is sensitive with respect to "flow number 3". This can be seen from the LES results were an overall good agreement is found for both meshes but a difference is seen in the region where "flow number 1" and "flow number 3" interacts.
For Model1_coarse "flow number 3" is stronger and higher velocity regions are extended further towards the cylinder head pushing "flow number 1" towards the cylinder head as well where it is found to be attached to the cylinder head further than for Model1_fine and the PIV results. Figure 6 confirms these observations as it shows that the "flow number 3" intensities are much stronger for Model1_coarse than for Model1_fine. It is an additional hint that Model1_ coarse is not adequate to capture the main flow features. Despite equal velocity magnitudes of "flow number 1" for both meshes this small difference in the tumble roll-up leads to a lag in the tumble front compared to the measurements as shown more clearly in Figure 7 for $190^{\circ}$ bTDC where the tumble flow has already performed nearly an entire revolution. The simulations nicely show how the tumble vortex is centered within the piston bowl at this timing. As a result the shape and position of the tumble is centered in the cylinder resulting in a very similar shape of the high velocity tumble flow for both meshes. The main difference is the tumble front location being $\sim 5 \mathrm{~mm}$ lower for 


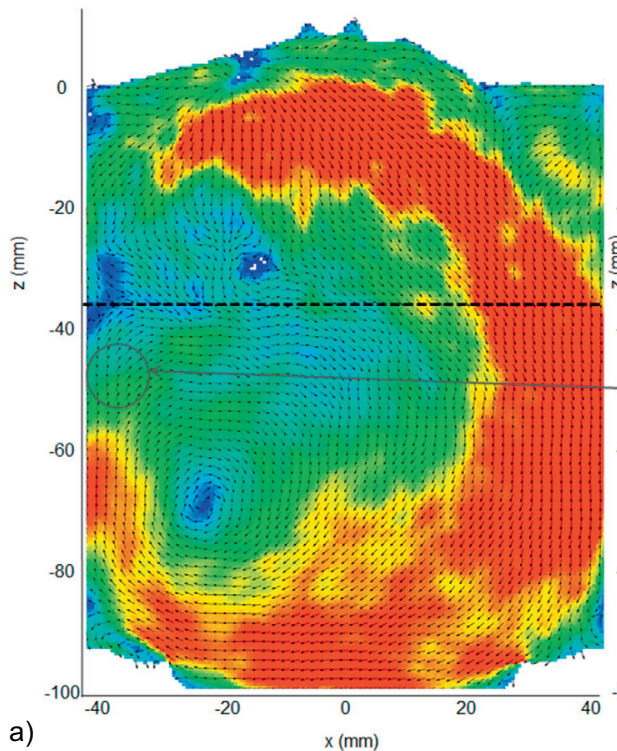

a) $\mathrm{x}(\mathrm{mm})$ b)

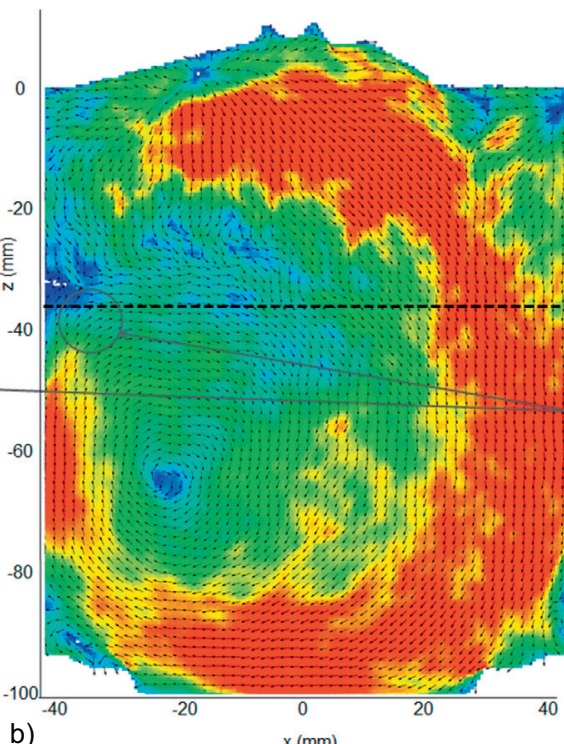

$\mathrm{x}(\mathrm{mm})$

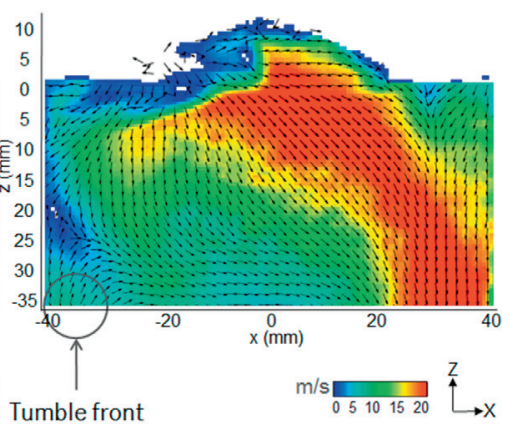

Figure 7

Average in-plane velocity magnitude $\left(V_{x z}\right)$ in the central tumble plane @ $190^{\circ}$ CA bTDC. LES results averaged over 12 cycles (a) Model1_coarse, b) Model1_fine). c) High-speed PIV results averaged over 200 cycles (Campaign1) every (3rd 4th) vector shown.

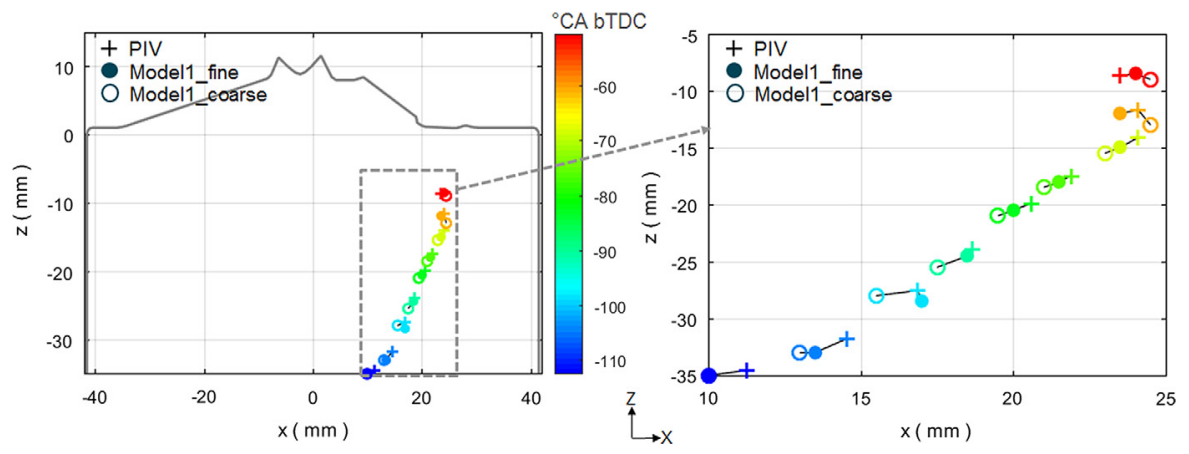

Figure 8

Tumble center evolution in high speed PIV (Campaign1) and LES (Model1 fine and Model1_coarse). LES results averaged over 12 cycles. High-speed PIV results averaged over 200 cycles.

Model1_coarse with a corresponding shift of the forming tumble vortex center.

Furthermore as the intake flow path starting from the airbox towards the plenum is positioned perpendicular to the central tumble plane of the engine (Fig. 3), the in-cylinder flow is expected to be slightly asymmetrical. Figure 6 shows that strong differences can be observed between the "flow number 3" intensities coming from both intake valves leading to a stronger asymmetrical flow in the cylinder with Model1_coarse compared to Model1 fine.

The evolution of the tumble flow during compression is further characterized by the tumble vortex center.
The $\Gamma$ criterion as proposed by Graftieaux [24] was used to determine the global center of rotation in a twodimensional flow region (i.e. tumble center for in-cylinder flows). At each position $p(x, y)$ in the domain, the angle $\gamma_{i}$ $(l, m)$ between any velocity vector at the position $(l, m)$ and its connection vector $p(x, y)$ is calculated. The $\Gamma$ criterion (1) is then applied for the $N$ velocity vectors present in the analyzed region. The global center of rotation is found were $\Gamma$ has the highest value.

$$
\Gamma(x, y)=\frac{1}{N} \sum_{i=1}^{N} \sin \gamma_{i}(l, m)
$$




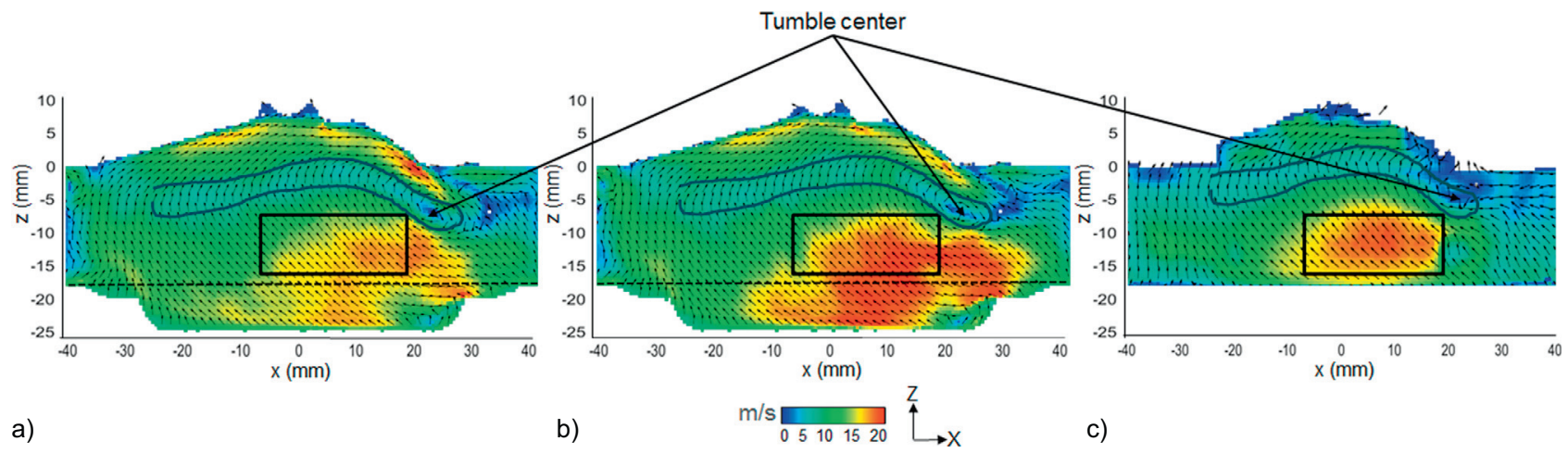

Figure 9

Average in-plane velocity magnitude $\left(V_{x z}\right)$ in the central tumble plane @ $40^{\circ}$ CA bTDC. LES results averaged over 12 cycles (a) Model1_coarse, b) Model1_fine), c) high-speed PIV results averaged over 200 cycles (Campaign1) every (3rd 4th) vector shown.

This criterion has been applied by Stiehl et al. [25] to high speed PIV results in order to compare two intake port geometries or by Janas et al. [13] to compare LES results with high speed PIV measurements for validation. Within this work the $\Gamma$ criteria was applied to the mean flow fields from 110 to $50^{\circ} \mathrm{CA}$ bTDC and shown in Figure 8 for the PIV measurements (represented by the crosses) and both LES (filled circles for Model1_fine and hollow circles for Model1_coarse). The same two-dimensional flow domain in the central tumble plane was used in LES and PIV. The evolution of the tumble center path from both simulations is in good agreement with the measurements with Model1_fine being somehow closer to the measurements. Differences of the averaged tumble location are in the order of $1 \mathrm{~mm}$. With the current engine geometry, the tumble center moves from the middle of the cylinder toward the exhaust valves until $70^{\circ} \mathrm{CA}$ bTDC. Further on its direction changes and becomes parallel to the cylinder $z$-axis.

The location of the tumble vortex is dominated by the piston bowl of the upwards moving piston and the location of its center becomes very similar for both simulations despite the observed differences early during intake. As discussed earlier small variations in the tumble roll-up leads to small differences of the tumble front location i.e. of the tumble flows rotational speed. This leads to a time shift of the appearance of the important "upward flow" below the spark plug as shown in Figure 9 for $40^{\circ} \mathrm{CA}$ bTDC. The flow structures are very similar in LES and PIV. One can distinguish the global tumble flow with its corresponding tumble center, the "upward flow" above the piston and the low velocity region in the vicinity of the tumble flow. While the flow structures and the tumble vortex centers are identical for both meshes the magnitude of the "upward flow" marked by the box is lower for Model1_coarse and is in good agreement with the measurements for Model1_fine. Even though the tumble vortex center trajectory is captured correctly by LES, this criterion is not sufficient for validation since the tumble center position is barely affected by the changes of the local flow structures. Therefore the visual observation of the local 2D flow structures together with the understanding of the flow interactions (flow number 1-4) cannot be replaced by the sole "tumble vortex center" validation criterion.

The creation of the "upward flow" cannot be explained only by the analysis of the flow in the central tumble plane. The out-of-plane velocity component was found to play a crucial role $[4,5]$ and should not be neglected when analyzing the LES. A further LES study of the origin of the upward flow combined with a dual-plane high speed PIV analysis is the subject of on-going work.

\subsection{Influence of the Spark Plug}

To investigate the influence of the spark plug on the flow field the LES was repeated using the optimized Model1_fine with the spark plug included. The resulting mesh is termed Model2. Figure 10 shows the mean flow field in the central tumble plane at $40^{\circ} \mathrm{CA}$ bTDC with and without the spark plug as captured by LES and PIV. In the presence of the spark plug the flow direction of the "upward flow" remains the same but at a reduced flow magnitude. This is captured well by the LES. Without the spark plug the tumble vortex centre is still visible close to the cylinder head in both the PIV and LES results. With the spark plug in place the tumble vortex is trapped in the corner between the spark plug and the cylinder head as shown by the LES. This is not captured by the PIV measurements due to the reduced signal quality in the vicinity of the spark plug caused by the reflections.

This analysis shows the influence of the spark plug on the in-cylinder flow. As expected the impact of the spark plug on the flow field was not only found locally but had an global 


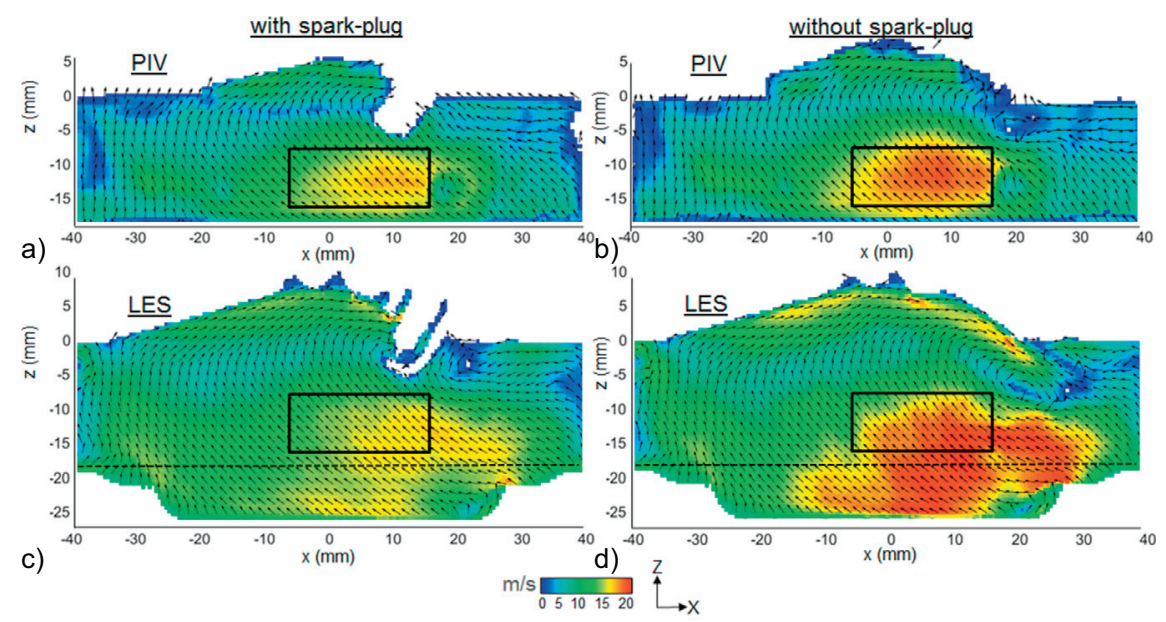

Figure 10

Average in-plane velocity magnitude $\left(V_{x z}\right)$ in the central tumble plane @ $40^{\circ}$ CA bTDC. Top: High-speed PIV results. a) Averaged over 300 cycles (Campaign2), b) averaged over 200 cycles (Campaign1). Bottom: LES results c) Averaged over 35 cycles (Model2), d) averaged over 12 cycles (Model1_fine).

effect on the flow field. Since the "upward flow" is of great importance for engine performance any change of this flow region due to simplifications of the computational domain by removing the spark plug would lead to erroneous results. This effect might be stronger within this configuration where the spark plug was found directly in the path of the tumble vortex center. However, the effect of the spark needs to be considered if data taken from measurements or simulations without the spark plug in place are used within engine development.

\subsection{Validation of the Cyclic Velocity Variations}

The aim of this work is to provide a validated LES methodology in order to study the cyclic variability of the "upward flow" which was originally identified by PIV measurements in Campaign2. Therefore it is important to understand how well the CCV are captured within the simulations. For this comparison the LES was performed on Model 2 with the spark plug in place capturing 35 individual cycles. For a quantitative comparison the ensemble averaged in-plane velocity was calculated from the region where the "upward flow" is found at $40^{\circ}$ bTDC defined by the inserted black box in Figure 10. Figure 11 shows a comparison of the ensemble averaged velocity magnitude distributions from LES and PIV at 60, 50 and $40^{\circ}$ CA bTDC, while the distance between the box and the piston is kept constant. The mean velocity in LES is always slightly shifted towards lower velocities. However the difference between LES and high speed PIV is in the range of $1-1.5 \mathrm{~m} / \mathrm{s}$.
Figure 12 shows a comparison of the mean velocity and standard deviation at 60,50 and $40^{\circ} \mathrm{CA}$ bTDC. Eight samples of 35 consecutive PIV cycles each are compared to the 35 LES cycles. The standard deviation is increasing from 60 to $40^{\circ} \mathrm{CA}$ bTDC for both, PIV and LES. The LES results are within the values obtained from the eight PIV samples. The standard deviation of the 35 LES cycles at $40^{\circ} \mathrm{CA}$ bTDC is approx. $40 \%$ lower than the one determined from the 300 PIV cycles.

The two sample Kolmogorov-Smirnov test, which can be applied to small sample sizes, was therefore used to check whether the two independent LES and PIV samples come from the same distribution. As null hypothesis, it is assumed that both samples originate from the same distribution. The cumulative distribution functions $Y_{\mathrm{LES}}(x)$ with a sample size $n=35$ and $Y_{\mathrm{PIV}}(x)$ with $m=300$ are compared and the test quantity $\bar{D}$ is defined as the maximum vertical deviation between the cumulative distributions of both samples:

$$
\bar{D}=\max \left|Y_{\mathrm{LES}}(x)-Y_{\mathrm{PIV}}(x)\right|
$$

$H_{0}$ is rejected as soon as $\bar{D}$ exceeds the critical value $D_{\alpha}$

$$
D_{\alpha}=K_{\alpha} \sqrt{\frac{n+m}{m n}}
$$

with $K_{\alpha}$ being the inverse of the Kolmogorov distribution taken from [26]. For a significance value $\alpha=0.05$ the critical value is $D_{0.05}=0.243$. Figure 13 shows the cumulative distributions of LES and PIV. The test quantity obtained $\bar{D}=0.195$ is below $D_{0.05}$ thus the null hypothesis is accepted and from a statistical point of view, the ensemble 

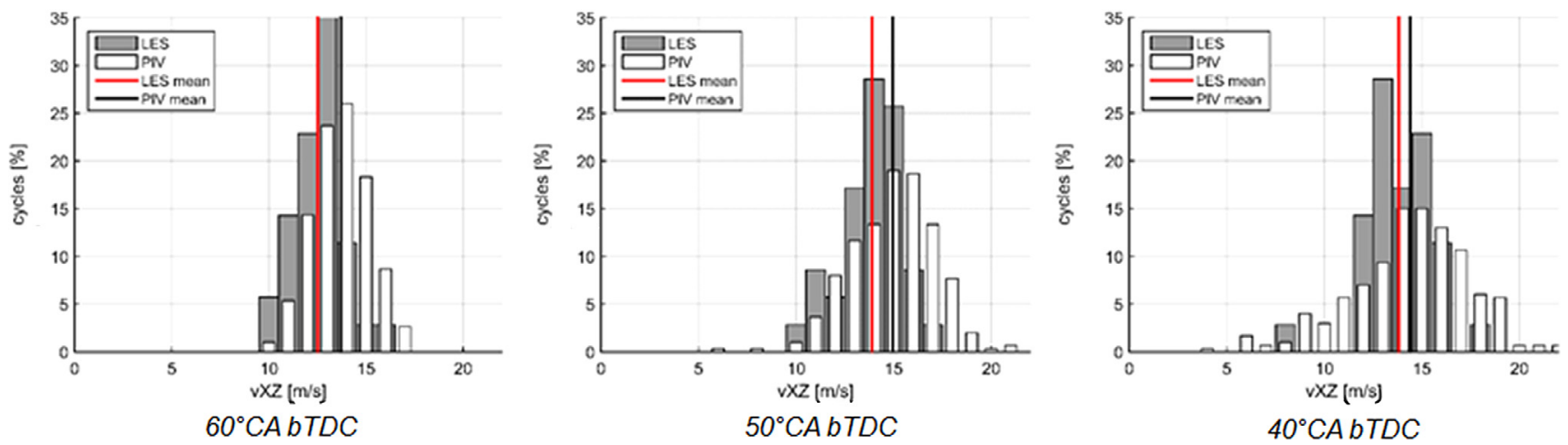

Figure 11

Ensemble averaged velocity distribution comparisons between LES (Model2) and high speed PIV (Campaign2).
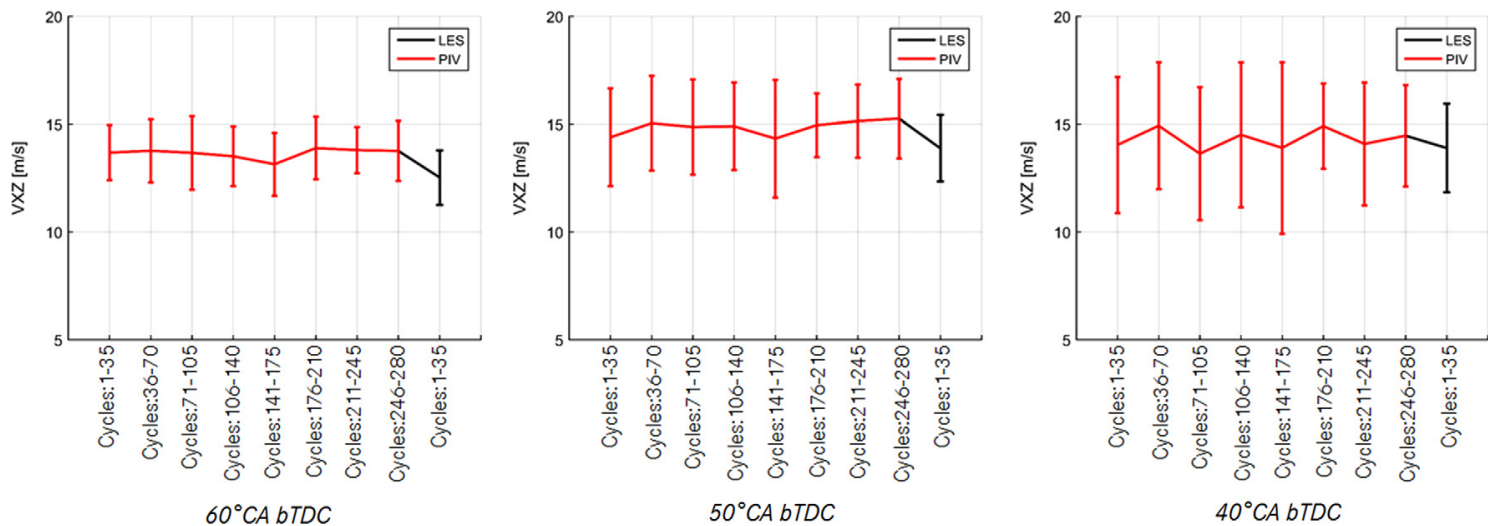

Figure 12

Mean velocity and standard deviation comparisons between LES (Model2) and high speed PIV (Campaign2) using samples of 35 cycles.

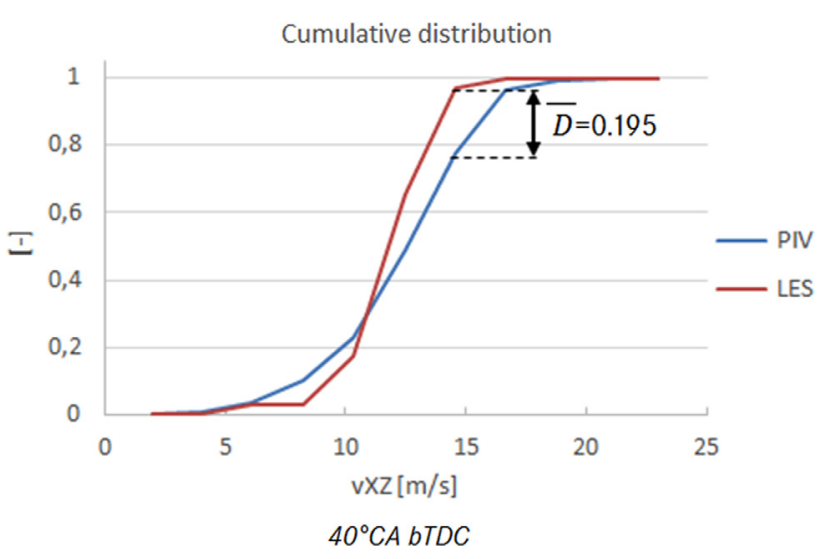

Figure 13

Comparison of the cumulative distribution of the ensemble averaged velocity between LES (Model2, 35 cycles) and high speed PIV (Campaign2, 300 cycles). averaged velocity magnitude distribution from LES is not significantly different to the one from PIV in the "upward flow" region before injection at $40^{\circ} \mathrm{CA}$ bTDC.

\section{CONCLUSION AND OUTLOOK}

This work comprised a combined experimental and numerical investigation of the in-cylinder flow in an optically accessible DISI-engine. The objective was to present a new LES validation strategy where high speed PIV has been used first to identify the relevant quantities and regions of the flow important for engine development and then validate the simulation by direct comparisons. An iterative process between high speed PIV and LES was put in place where an extensive mesh study allowed to converge towards a robust CFD model reproducing the experimental results during the intake and compression strokes. This study has shown the necessity 
of using a standard post-processing tool to analyze and compare identically the LES and high speed PIV results together. An engine geometry variation in LES confirmed the robustness of the meshing set-up found in this study.

The LES with AVBP has proved its ability to capture very well both the mean in-cylinder flow and the cyclic variability of the flow velocity in the central tumble plane. The cyclic variability of the "upward flow" which was identified in previous work to be responsible for $\mathrm{CCV}$ of the stratified combustion mode [4] was well captured in LES and successfully validated with the PIV measurements. A further LES study on the formation of the "upward flow" combined with a dual-plane high speed PIV analysis is the subject of on-going work.

\section{REFERENCES}

1 Lückert P., Breitbach H., Waltner A., Merdes N., Weller R (2011) Potentials of spray-guided combustion systems in combination with downsizing concepts, in 32nd International Vienna Motor Symposium 2011, 5-6 May, Vienna.

2 Vent G., Enderle C., Merdes N., Kreitmann F., Weller R. (2012) The new 2.0 1 Turbo engine from the Mercedes-Benz 4-cylinder engine family, in 21st Aachen Colloquium Automobile and Engine Technology.

3 Bode J., Schorr J., Krüger C., Dreizler A., Böhm B. (2017) Influence of three-dimensional in-cylinder flows on cycle-tocycle variations in a fired stratified DISI engine measured by time-resolved dual-plane PIV, Proc. Combust. Inst. 36, 3, 3477-3485.

4 Krüger C., Schorr J., Nicollet F., Bode J., Dreizler A., Böhm B. (2017) Cause-and-effect chain from flow and spray to heat release during lean gasoline combustion operation using conditional statistics, Int. J. Engine Res. 18, 143-154.

5 Bode J., Krüger C., Schorr J., Dreizler A., Böhm B. (2016) Influence of three-dimensional in-cylinder flows on cycle-to-cycle variations in a fired stratified DISI engine measured by timeresolved dual-plane PIV. Proc. Combust. Inst. 36, 3, 3477-3485.

6 Boree J., Miles PC (2014) In-cylinder flow, in: Crolla D, Foster D.E., Kobayashi T., Vaughan N. (eds), Encyclopedia of automotive engineering, John Wiley \& Sons, Chichester, UK, pp. 1-31.

7 Baum E., Peterson B., Böhm B., Dreizler A. (2014) On the validation of LES applied to internal combustion engine flows: part 1: comprehensive experimental database, Flow Turbul. Combust. 92, 1-2, 269-297.

8 Buschbeck M., Bittner N., Halfmann T., Arndt S. (2012) Dependence of combustion dynamics in a gasoline engine upon the in-cylinder flow field, determined by high-speed PIV, Exp. Fluids. 53, 6, 1701-1712.

9 Peterson B., Reuss D., Sick V. (2014) On the ignition and flame development in a spray-guided direct-injection spark-ignition engine, Combust. Flame 161, 1, 240-255.

10 Stiehl R., Bode J., Schorr J., Krüger C., Dreizler A., Böhm B. (2016) Influence of intake geometry variations on in-cylinder flow and flow-spray interactions in a stratified direct-injection spark-ignition engine captured by time-resolved particle image velocimetry, Int. J. Engine Res. 17, 9, 983-997.
11 Baum E., Peterson B., Surmann C., Michaelis D., Böhm B., Dreizler A. (2013) Investigation of the 3D flow field in an IC engine using tomographic PIV, Proc. Combust. Inst. 34, 2, 2903-2910.

12 Haworth D. (1999) Large-eddy simulation of in-cylinder flows, Oil Gas Sci. Technol. - Rev. IFP 54, 2, 175-185.

13 Janas P., Wlaukas I., Böhm B., Kempf A. (2016) On the evolution of the flow field in a spark ignition engine, Springer Science+Business Media, Dordrecht.

14 Baumann M., Di Mare F., Janicka J. (2014) On the validation of large eddy simulation applied to internal combustion engine flows part II: numerical analysis, Flow Turbul. Combust. 92, 1-2, 299-317.

15 Robert A., Richard S., Colin O., Martinez L., De Francqueville L. (2014) LES prediction and analysis of knocking combustion in a spark ignition engine, Proc. Combust. Inst. 35, 3, 2941-2948.

16 Robert A., Richard S., Colin O., Poinsot T. (2015) LES study of deflagration to detonation mechanisms in a downsized spark ignition engine, Combust. Flame 162, 7, 2788-2807.

17 Iafrate N. (2016) Simulation aux grandes échelles diphasique dans les moteurs downsizes à allumage commandé, $P h D$, Université de Toulouse.

18 Goryntsev D., Sadiki A., Janicka J. (2011) Towards large eddy simulation of spray combustion in direct injection spark ignition engine, SAE Technical Paper 2011-01-1884.

19 Abraham P., Liu K., Haworth D., Reuss D., Sick V. (2014) Evaluating large-eddy simulation (LES) and high-speed particle image velocimetry (PIV) with phase-invariant proper orthogonal decomposition (POD), Oil Gas Sci. Technol. Rev. IFP 69, 1, 41-59.

20 Hasse C. (2015) Scale-resolving simulations in engine combustion process design based on a systematic approach for model development, Int. J. Engine Res. 17, 1, 44-62.

21 AVBP: Property of CERFACS. Website: http://www.cerfacs.fr/ $\operatorname{avbp} 7 \mathrm{x} /$.

22 Nicoud E., Colin O., Angelberger C., Krüger C., Nicollet F. (2016) A no-slip wall law formulation for cell-vertex codes, validated for LES of internal aerodynamics, in LES4ICE Conference 2016, IFPEN, Rueil-Malmaison.

23 Freudenhammer D., Baum E., Peterson B., Böhm B., Grundmann S. (2014) Towards time-resolved magnetic resonance velocimetry for IC-engine intake flows, Exp. Fluids. $\mathbf{5 5}, 1724$.

24 Graftieaux L., Michard M., Grosjean N. (2001) Combining PIV, POD and vortex identification algorithms for the study of unsteady turbulent swirling flows, Meas. Sci. Technol. 12, 9, 1422.

25 Stiehl R., Schorr J., Krüger C., Dreizler A., Böhm B. (2013) In-cylinder flow and fuel spray interactions in a stratified spray-guided gasoline engine investigated by high-speed laser imaging techniques, Flow Turbul. Combust. 91, 431-450.

26 Sachs L. (1984) Applied statistics a handbook of techniques, Springer-Verlag, New York.

Manuscript submitted in April 2017 Manuscript accepted in June 2017 Published online in September 2017 